\title{
An Attempt to Use Weighted Cusums to Identify Sublanguages
}

\author{
Harold Somers \\ Centre for Computational Linguistics, UMIST \\ PO Box 88, Manchester M60 1QD \\ England \\ harold@ccl.umist.ac.uk
}

\begin{abstract}
This paper explores the use of weighted cusums, a technique found in authorship attribution studies, for the purpose of identifying sublanguages. The technique, and its relation to standard cusums (cumulative sum charts) is first described, and the formulae for calculations given in detail. The technique compares texts by testing for the incidence of linguistic 'features' of a superficial nature, e.g. proportion of 2- and 3-letter words, words beginning with a vowel, and so on, and measures whether two texts differ significantly in respect of these features. The paper describes an experiment in which 14 groups of three texts each representing different sublanguages are compared with each other using the technique. The texts are first compared within each group to establish that the technique can identify the groups as being homogeneous. The texts are then compared with each other, and the results analysed. Taking the average of seven different tests, the technique is able to distinguish the sublanguages in only $43 \%$ of the case. But if the best score is taken, $79 \%$ of pairings can be distinguished. This is a better result, and the test seems able to quantify the difference between sublanguages.
\end{abstract}

Keywords: sublanguage, genre, register, weighted cusum.

\section{Introduction}

This paper concerns a technique which we use to measure whether two texts are representative of the same text genre or sublanguage. It is very much in the spirit of the well-known work in this field by Douglas Biber (1988, 1990, 1995), but differs crucially in that we avoid the explicit selection of linguistic features thought a priori likely to be important in distinguishing sublanguages, and instead use a set of low-level features based on trivial aspects of the words such as length and initial letter. Our technique is borrowed from the neighbouring field of authorship attribution (for an overview of this field see Ule 1982; Smith 1982; Potter 1991; Burrows 1992; Holmes 1994). It is a straightforward calculation, simple to implement, and very general in application. It can be used with fairly small texts. This paper describes an experiment to see whether the technique can be used for the sublanguage identification task, even though it was originally designed for a somewhat different problem.

In Somers (forthcoming), we used a technique called 'weighted cusums' to investigate how well a parody of Lewis Carroll had imitated his style. Looking also at other writings by Carroll, including his 'serious' mathematical works (under his real name, Charles Dodgson), letters to adults and children, his diaries, formal and whimsical articles in newspapers, we found that the technique, although unable to identify Carroll/Dodgson as the unique author of all the texts, as the authorship attribution literature would demand and expect, seemed to be able to group together his writings according to genre and/or topic. This was an interesting finding, because the technique, as has already been hinted, measures the most banal of linguistic features. This finding suggested to us the idea of the experiment reported in this paper: could the technique be used to identify sublanguages?

\section{Background}

\subsection{Sublanguage}

We will assume that readers of this paper are fairly familiar with the literature on sublanguage (e.g. Kittredge \& Lehrberger 1982; Grishman \& Kittredge 1986), including definitions of the notion, history of the basic idea, and, above all, why it is a useful concept. Some readers will prefer terms like 'register' (which Biber uses); an affinity with work on genre detection will also be apparent. Because there is sometimes some dispute about the use of the term 'sublanguage', let us clarify from the start that for our purposes a sublanguage is an identifiable genre or text-type in a given subject field, with a relatively or even absolutely closed set of syntactic structures and vocabulary. In recent years, the availability of large corpora and 'new' methods to process them have led to renewed interest in the question of sublanguage identification (e.g. Sekine 1997), while Karlgren \& Cutting (1994) and Kessler et al. (1997) have focussed on the narrower but clearly related question of genre.

Our purpose in this paper is to explore a technique for identifying whether a set of texts 'belong to' the 
same sublanguage, and of quantifying the difference between texts: our technique compares texts pairwise and delivers a 'score' which can be used to group texts judged similar by the technique. As we shall see later, what is of interest here is that the score is derived from a simple count of linguistic features such as word length and whether words begin with a vowel; yet this apparently unpromising approach seems to deliver usable results.

In his well-known study, Biber (1988) took a number of potentially distinct text genres and measured the incidence of 67 different linguistic features in the texts to see what correlation there was between genre and linguistic feature. He also performed factor analysis on the features to see how they could be grouped, and thereby see if sublanguages could be defined in terms of these factors.

The linguistic features that Biber used ${ }^{l}$ are a mixture of lexical and syntactic ones, and almost all require a quite sophisticated level of analysis of the text data - dictionary look-up, tagging, a parser. They are presumably also, it should be said, hand-picked as features whose use might differ significantly from one genre to another. Although Biber gives details of the algorithms used to extract the features, it is not a trivial matter to replicate his experiments.

Kessler et al. (1997) make the same criticism of Biber and of Karlgren \& Cutting (1994), and restrict their experimentation on genre recognition to "surface cues". In their paper they do not give any detail about the cues they use, except to say that they are "mainly punctuation cues and other separators and delimiters used to mark text categories like phrases, clauses, and sentences" (p. 34); however, Hinrich Schütze (personal communication) has elaborated that "The cues are punctuation, non-content words (pronouns, prepositions, auxiliaries), counts of words, [of] unique words, [of] sentences, and [of] characters; and deviation features (standard deviation of word length and sentence length)". As we shall see below, the use of superficial linguistic aspects of the text is a feature of the approach described here.

\subsection{Authorship attribution and weighted cusums}

Authorship attribution has for a long time been a significant part of literary stylistics, familiar even to lay people in questions such as "Did Shakespeare really write all of his plays?", "Who wrote the Bible?", and

The features can be grouped into "sixteen major categories: (A) tense and aspect markers, (B) place and time adverbials, $(C)$ pronouns and pro-verbs, (D) questions, (E) nominal forms, (F) passives, (G) stative forms, $(\mathrm{H})$ subordination feanures, (I) adjectives and adverbs, (J) lexical specificity, (K) specialized lexical classes, (L) modals, (M) specialized verb classes, $(N)$ reduced or dispreferred forms, $(O)$ coordination, and (P) negation." (Biber 1988:223) so on. With the advent of computers, this once rather subjective field of study has become more rigorous, attracting also the attention of statisticians, so that now the field of 'stylometrics' - the objective measurement of (aspects) of literary style - has become a precise and technical science.

One technique that has been used in authorship attribution studies, though not without controversy, is the cumulative sum chart ('cusum') technique, a variant of which we shall be using for our own investigation. Since we are not actually using standard cusums here, our explanation can be relatively brief. Cusums are a fairly well-known statistical device used in process control. The technique was adapted for author identifcation by Morton (1978) - see also Farringdon (1996) - and achieved some notoriety for its use in court cases (e.g. to identify faked or coerced confessions) as well as in literary studies. The technique is easy to implement, and requires only small amounts of text.

A cusum is a graphic plot based on a sequence of measures. For example, suppose we have a set of measures $\langle 11,7,4,10,2, \ldots\rangle$ with a mean value of 6. The corresponding divergences from the mean are $\langle 5,1,-2,4,-4, \ldots\rangle$. The cusum chart plots not these divergences, but their aggregate sum, i.e. $\langle 5,6,4,8,4, \ldots\rangle$, the sequence inevitably ending in 0 . The plot reflects the variability of the measure: the straighter the line, the more stable the measure. In authorship attribution studies, the cusum chart is used to plot the homogeneity of a text with respect to a linguistic 'feature' such as use of two- and three-letter words on a sentence-by-sentence basis. Two graphs are plotted, one for the sentence lengths, the other for the incidence of the feature, and superimposed after scaling so that they cover roughly the same range. The authorship identification technique involves taking the texts in question, concatenating them, and then plotting the cusum chart. If the authors differ in their use of the linguistic feature chosen, this will manifest itself as a marked divergence in the two plots at or near the point(s) where the texts have been joined.

There are a number of drawbacks with this method, the main one being the manner in which the result of the test is arrived at, namely the need to scrutinize the plot and use one's skill and experience (i.e. subjective judgment) to determine whether there is a "significant discrepancy" at or near the join point in the plot.

A solution to this and several other problems with the standard cusum technique is offered by Hilton \& Holmes (1993) and Bissell (1995a,b) in the form of weighted cusums (henceforth WQsums). Since this is the technique we shall use for our experiments, we need to describe it in full detail. 


\section{Weighted cusums}

\subsection{The calculations}

As in the standard cusum, the WQsum is a measure of the variation and homogeneity of use of a linguistic feature on a sentence-by-sentence basis throughout a text. It captures not only the relative amount of use of the feature, but also whether its use is spread evenly throughout the texts in question.

In a WQsum, instead of summing the divergence from the mean $w_{i}-\bar{w}$ for the sentence lengths $w$ and similarly $x_{i}-\bar{x}$ for the linguistic feature $x$, we sum $x_{i}-$ $\hat{\pi} w_{i}$, where $\hat{\pi}$, the 'weight', is the overall proportion of feature words in the whole text, as given by (1). As Hilton \& Holmes (1993) explain, this weighting means that we are calculating "the cumulative sum of the difference between the observed number of feature occurrences and the 'expected' number of occurrences" (p. 75).

$$
\hat{\pi}=\frac{\sum x_{i}}{\sum w_{i}}
$$

As we shall see shortly, the variation in a WQsum can be measured systematically, and its statistical significance quantified with something like a $t$-test. This means that visual inspection of the WQsum plot is not necessary. There is no need, either, to concatenate or sandwich the texts to be compared. For the $t$-test, the two texts, $A$ and $B$, are treated as separate samples. The formula for $t$ is (2).

$$
t=\frac{\left|\hat{\pi}_{A}-\hat{\pi}_{B}\right|}{\sqrt{\left.\sum \hat{\sigma}_{A}\right)^{2}}+\frac{\left(\dot{\sigma}_{B}\right)^{2}}{\sum w_{B}}}
$$

The $t$-value is, in the words of Hilton \& Holmes, "a measure of the evidence against the null hypothesis that the frequency of usage of the habit [i.e. linguistic feature] under consideration is the same in Text $A$ and Text $B$. The higher the $t$-value, the more evidence against the hypothesis" (p. 76). The formula chosen for the calculation of variance $\hat{\sigma}$ in (2) is given in (3), where $n$ is the number of sentences in the text.

$$
\hat{\sigma}^{2}=\frac{1}{n-1} \sum_{i=1}^{n-1} \frac{\left(\frac{x_{i}}{w_{i}}-\frac{x_{i+1}}{w_{i+1}}\right)^{2}}{\left(\frac{1}{w_{i}}+\frac{1}{w_{i+1}}\right)}
$$

The resulting value is looked up in a standard $t$ table, which will tell us how confidently we can assert that the difference is significant. For this we need to know the degrees of freedom $v$, which depends on the number of sentences in the respective texts, and is given by (4). Tradition suggests that $p<.05$ is the minimum acceptable confidence level, i.e. the probability is less than $5 \%$ that the differences between the texts are due to chance.

$$
v=n_{A}+n_{B}-2
$$

\subsection{The linguistic features}

A point of interest for us is that both the cusums and WQsums have been used in the stylometrics field to measure the incidence of linguistically banal features, easily measured and counted. The linguistic features proposed by Farringdon (1996:25), and used in this experiment, involve the number of words of a given length, and/or beginning with a vowel, as listed in Table

\begin{tabular}{|c|c|}
\hline Habit & Abbreviation \\
\hline Two- and three-letter words & lw23 \\
\hline Two-, three- and four-letter words & lw234 \\
\hline Three- and four-letter words & lw34 \\
\hline Initial-vowel words & vowel \\
\hline $\begin{array}{l}\text { Two- and three-letter words or } \\
\text { initial-vowel words }\end{array}$ & $1 \mathrm{w} 23 \mathrm{v}$ \\
\hline $\begin{array}{l}\text { Two-, three- and four-letter words or } \\
\text { initial-vowel words }\end{array}$ & $1 w 234 v$ \\
\hline $\begin{array}{l}\text { Three- and four-letter words or } \\
\text { initial-vowel words }\end{array}$ & $\operatorname{lw} 34 \mathrm{v}$ \\
\hline
\end{tabular}
1.

Table 1 Linguistic features identified by Farringdon (1996:25).

Other experimenters have suggested counting the number of nouns and other parts of speech, but it is not clear if there are any limitations on the linguistic features that could be used for this test, except the obvious one that the feature should in principle be roughly correlated with sentence length. In any case, part of the attraction for our experiment is that the features are so fundamentally different from the linguistic features used by Biber in his experiments, and so will offer a point of comparison. Furthermore, they are easy to compute and involve no overheads (lexicons, parsers etc.) whatsoever.

It is also interesting to note that the WQsum is a measure of variation, a type of metric which, according to Kessler et al. (1997) has not previously used in this type of study.

In authorship identification, it is necessary first to determine which of these features is "distinctive" for a given author, and then to test the documents in question for that feature. This is not appropriate for our sublanguage experiment, so for each text comparison we run all seven tests. Each test gives us a $t$-score from which a confidence level can be determined. Obviously, the result over the seven tests may vary somewhat. For our experiment we simply take the average of the seven $t$-scores as the result of text comparison. It 
is not obvious that it makes sense any more to treat this as a $t$-score, and in the experiments described below we tend to treat it as a raw score, a lower score indicating cohesion, a higher score suggesting difference. Nevertheless it is useful to bear in mind that, given the degrees of freedom involved in all cases (the texts are all roughly the same length), the threshold for significance is around 1.65 .

\section{The method}

Our experiment is to use the WQsum test on a corpus of small texts which we believe can be grouped according to genre or sublanguage. We gathered 15 sets of different text-types: each set of three texts is assumed to represent a different sublanguage, and each text was written, as far as we know, by a different author. The 15 groups of texts were as follows:

blurbs publishers' announcements of scientific textbooks

BMJ abstracts of articles appearing in the British Medical Journal

childrens extracts from children's stories

church articles from local Catholic church newsletters

economy economic reports from a Swiss bank

e-mails discussing arrangement of a meeting

footie reports of soccer matches from the same newspaper, same date

lawreps extracts from The Weekly Law Reports

obits obituaries of Jacques Cousteau, from different newspapers

recipes recipes from the Internet Chef web site

TVscripts Autocue scripts from Central TV News programmes

tourism extracts from the "Shopping" section of Berlitz guides

univs descriptions of Computer Science courses

weather state-wide general weather forecasts from US National Weather Service

xwords sets of clues to cryptic crosswords

Our first task is to see that the WQsum test can confirm the homogeneity of the text triplets. For each group of three texts, we ran our test and averaged the $t$-scores for each group. Table 2 shows an example of this for the 'church' group of texts. Table 3 lists the 14 groups together with some information about the texts, including their 'homogeneity score', an indication of their length (average number of sentences, and average words per sentence), and their source.

The first thing to note is that all the groups of texts are well within the 1.65 threshold of significant difference. In other words, the pairwise WQsum test for each group firmly indicates homogeneity within the groups.
Table 2 WQsum test results for 'church' text set. Scores marked '*' suggest a difference significant at $p<.05$.

\begin{tabular}{lcccc}
\hline & A-B & A-C & B-C & overall \\
\hline lw23 & 0.576 & 0.388 & 0.055 & \\
lw234 & 0.131 & 0.781 & 0.834 & \\
lw34 & 0.906 & 0.102 & 0.843 & \\
vowel & $1.860^{*}$ & $1.729 *$ & 0.489 & \\
lw23v & 0.256 & 0.402 & 0.502 & \\
lw234v & 0.569 & 1.211 & 0.963 & \\
lw34v & 0.301 & 0.845 & 0.683 & \\
\hline av'ge & 0.657 & 0.780 & 0.624 & 0.687 \\
\hline
\end{tabular}

Table 3 The 15 genres, in order of 'homogeneity'.

The texts marked WWW were taken from the web, BNC the British National Corpus, and ECl the ACL/ECl CD-rom. Other texts are from my personal archive.

\begin{tabular}{llccr}
\hline Group & Source & Score & \multicolumn{2}{c}{ Av'ge length } \\
& & & sent & words \\
\hline obits & WWW & 0.440 & 25.67 & 19.10 \\
lawreps & BNC & 0.543 & 17.00 & 22.53 \\
emails & & 0.633 & 11.00 & 16.15 \\
univs & WWW & 0.659 & 21.33 & 24.87 \\
church & BNC & 0.687 & 18.00 & 19.39 \\
xwords & & 0.696 & 29.67 & 7.11 \\
TVscripts & BNC & 0.755 & 18.00 & 14.88 \\
BMJ & BNC & 0.802 & 19.00 & 17.19 \\
economy & ECI & 0.889 & 19.33 & 20.50 \\
weather & WWW & 0.890 & 24.33 & 9.69 \\
recipes & WWW & 0.976 & 26.00 & 7.68 \\
tourism & & 0.987 & 27.33 & 18.22 \\
blurbs & WWW & 1.083 & 11.67 & 23.00 \\
childrens & BNC & 1.174 & 26.00 & 11.99 \\
footie & WWW & 1.175 & 19.00 & 35.59 \\
\hline
\end{tabular}

We now proceed to compare all the texts with each other, pairwise. It is fortunate that the WQsum procedure is so simple, since this pairwise comparison involves a huge number of iterations: each text comparison involves seven applications of the WQsum test, each group comparison involves nine text comparisons, and there are 105 pairwise group comparisons, making a total of 6615 tests. In the following section we will attempt to summarize the findings to be had from this large body of data.

\section{Results}

The full results of the comparison are given in Table 4. This table shows the pairwise average $t$-scores, replicated for ease of consultation. The groups are ordered as in Table 3, so that results in the top left-hand corner 
of the table are between the most homogeneous groups, results in the bottom right the least homogeneous. The scores given on the diagonal are repeated from Table 3 and show the average score for the internal comparison of the texts in that group.

This time we are looking for high scores to support the hypothesis that the WQsum test can identify the texts as belonging to different sublanguages. At first glance the results look disappointing. If we again take a score of 1.65 as the notional cut-off point, then only $43 \%$ ( 45 out of 105 ) of the results qualify. On the other hand, if we compare the scores with those for the group-internal comparisons (Table 3 ), we may view the results more positively. The average internal score was 0.885 (s.d. $=0.232$ ), the worst score $1.175 ; 67 \%$ of our scores are better than that.

One problem stems from averaging the scores for all the tests. When the WQsum test is used in authorship attribution, it is necessary first to determine which linguistic feature is significant for the author under investigation. Looking at the raw scores for our experiment, we see that very often consistently high scores with one test are undermined by low scores on others. Table 5 shows an example of this, where an average score of 2.197 on the 'lw34' test is mitigated by insignificant scores on the other test, giving an overall average of 1.074 .

Table 5 Raw scores for 'childrens'-'emails' comparison.

\begin{tabular}{llllllllll}
\hline & lw23 & lw234 & lw34 & vowel & lw23v & lw234vlw34v \\
\hline A-X & 2.322 & 2.596 & 3.397 & 2.660 & 0.174 & 0.737 & 0.299 \\
A-Y & 0.732 & 1.896 & 3.725 & 0.796 & 0.261 & 1.460 & 1.205 \\
A-Z & 0.932 & 1.253 & 1.684 & 1.359 & 0.237 & 0.383 & 0.190 \\
B-X & 0.085 & 1.062 & 2.822 & 2.271 & 0.900 & 0.129 & 0.041 \\
B-Y & 1.732 & 0.714 & 3.090 & 0.941 & 1.215 & 0.553 & 0.622 \\
B-Z & 0.765 & 0.328 & 1.633 & 1.349 & 0.927 & 0.070 & 0.138 \\
C-X & 0.648 & 0.604 & 1.460 & 3.553 & 0.342 & 0.133 & 0.522 \\
C-Y & 1.420 & 0.169 & 1.795 & 1.449 & 0.692 & 0.419 & 0.455 \\
C-Z & 0.314 & 0.310 & 0.171 & 2.058 & 0.343 & 0.381 & 0.714 \\
\hline av'ge & 0.994 & 0.992 & 2.197 & 1.826 & 0.565 & 0.473 & 0.465 \\
\hline
\end{tabular}

So an alternative that suggests itself is to take in each case the highest of the average scores for each linguistic feature, on a pairwise basis. These alternative results are presented in Table 6 , which also shows in each case which linguistic feature gave the best result. Since we are now taking the highest rather than the average score for the pairwise comparisons, we should also take the highest score for within-group comparison, which is again shown on the diagonal. As in Table 4, the groups are ordered from 'best' to 'worst' withingroup score.
The 'improvement' in the results is considerable: this time 82 of the 105 results $(78 \%)$ are above the 1.650 threshold. However, taking the highest rather than the average score for the within-groups comparison leaves four of the groups - 'TVscripts', 'recipes', 'tourism' and 'childrens' - with scores above the 1.65 threshold, and a fifth group, 'weather', has a score very close to this. The scores for these groups are often high for comparisons with other texts, but they are also high for the within-group comparison: this suggests that the texts in these groups are not homogeneous, so we have to take this into account when we consider the results in the discussion that follows.

\section{Discussion}

\subsection{Does the WQsum test identify different sublanguages?}

Let us consider first the results as shown in Table 6 . Our main concern of course is to see whether the WQsum test can identify and distinguish the groups. Taking 1.65 as our threshold, we can rank the groups according to the number of other groups with which each gets a pairwise score average above this threshold. In addition, since the 'ideal' situation as far as our hypothesis goes would be for a low within-group average score suggesting homogeneity, and a high average score for comparisons with other groups, suggesting distinctiveness, as a further, informal measure of the extent to which the groups meet this condition, we can divide the average comparison score by the withingroup score. Table 7 shows a ranking of the groups along these lines.

The groups seem to divide into roughly four types. The first type, groups which support our hypothesis the best, have a low within-group average, a high pairwise average, and can easily be distinguished from most of the other groups. In this group are 'xwords', 'univs', 'blurbs', 'BMJ', and 'economy'. At the other end of the scale, at the bottom of Table 7, are those groups which can have a low 'Sig.' score. This group is not necessarily marked by a low pairwise average or a high within-group score: the 'obits' group for example has the second lowest within-group average, and scores quite highly on our informal ratio score. Yet the WQsum test cannot distinguish it from six of the other groups.

A third type is where the 'Sig.' score is high despite a high within-group average which would suggest lack of homogeneity. The 'recipes' group, for example, stands out as a distinct sublanguage, with highly significant scores compared to all other groups. Despite the fact that the within-group score is above the 


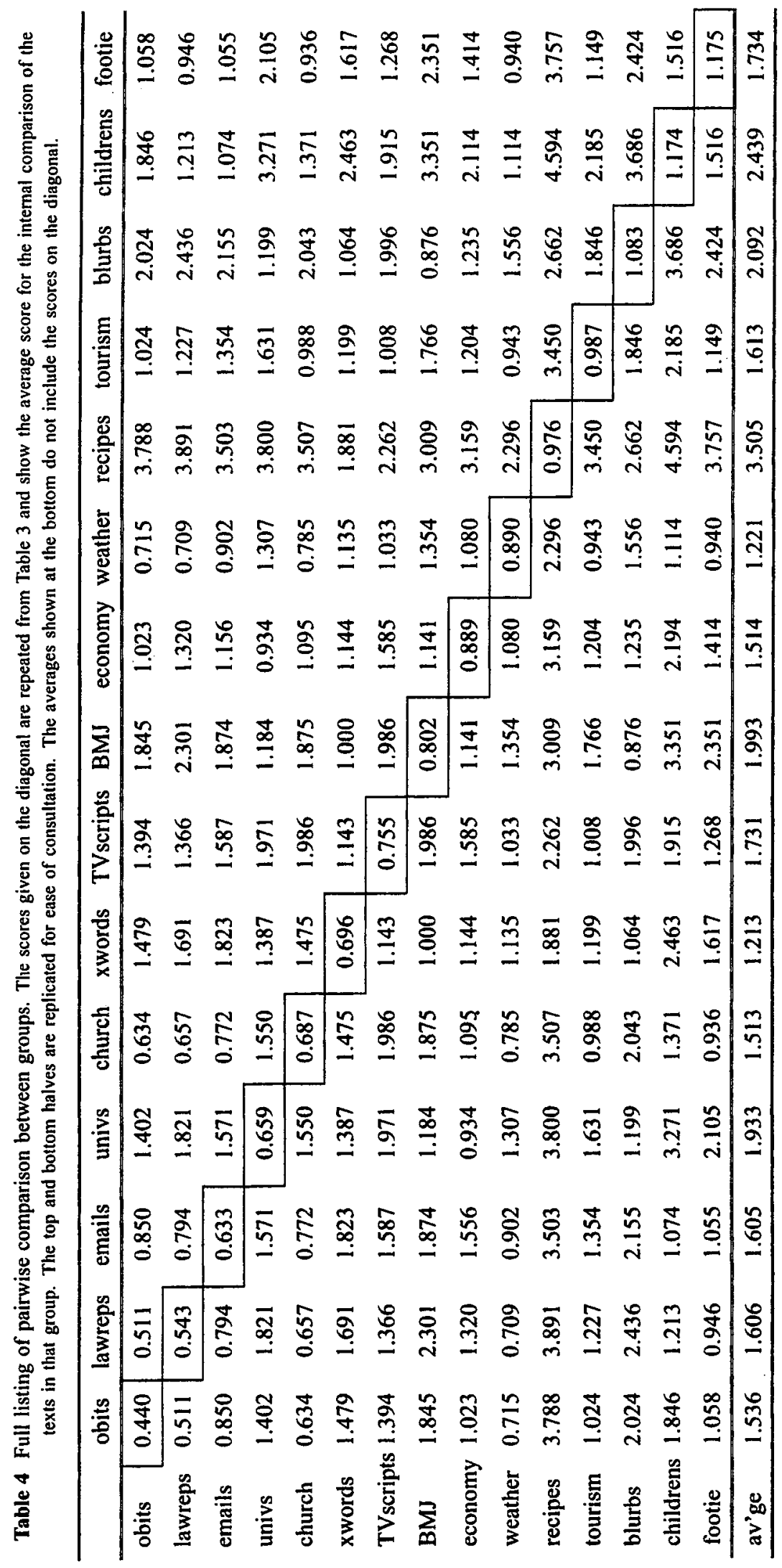




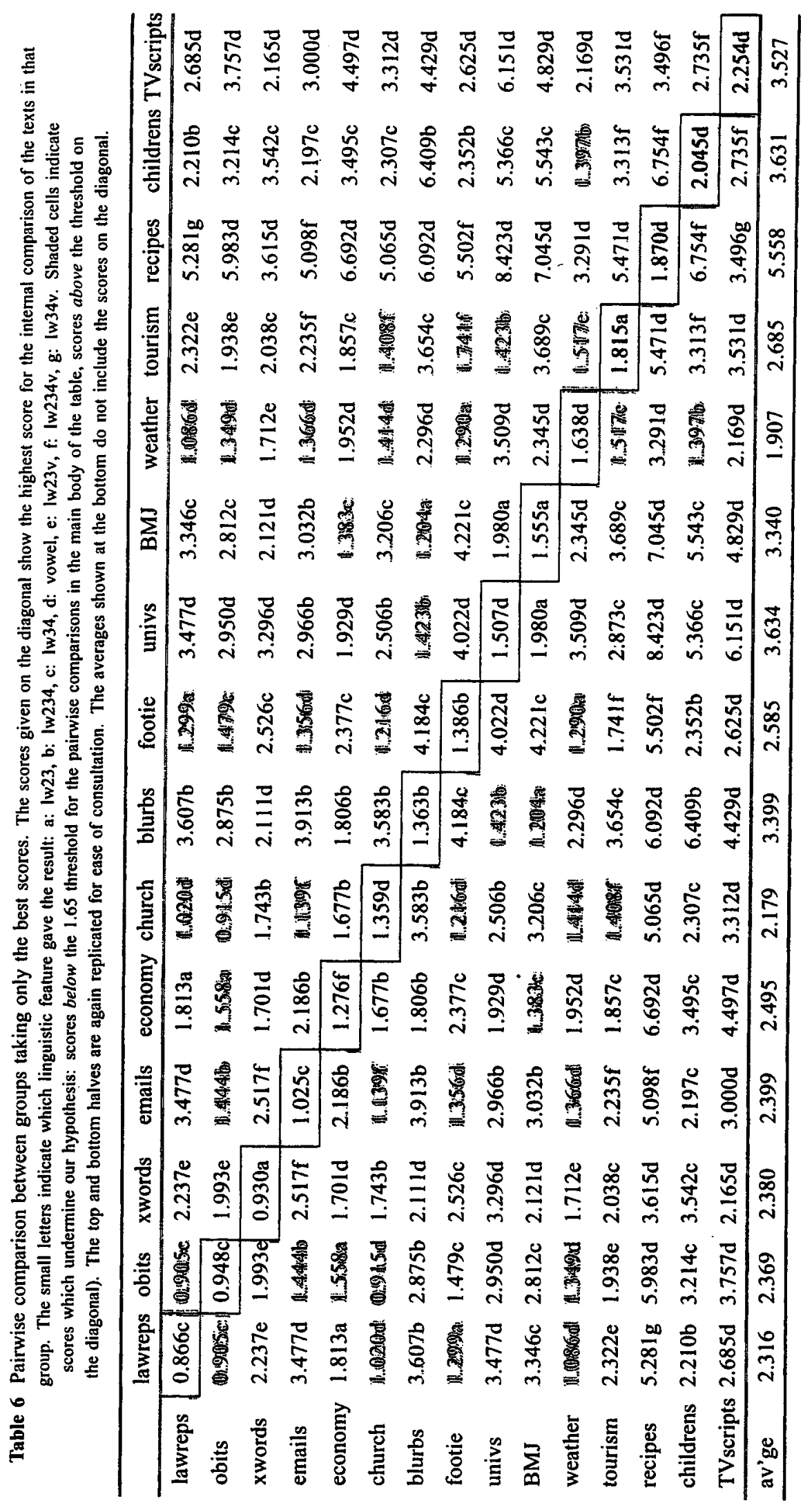


Table 7 The 15 groups ranked first according to total of pairwise averages above the 1.65 threshold ('Sig.'), and secondly according to the informal score described in the text. An asterisk indicates a group where the within-group average is above the 1.65 threshold.

\begin{tabular}{|c|c|c|c|c|c|}
\hline Group & & $\begin{array}{l}\text { Pairwise } \\
\text { average }\end{array}$ & $\begin{array}{l}\text { Within } \\
\text { group }\end{array}$ & Score & Sig. \\
\hline recipes & * & 5.558 & 1.870 & 2.972 & 14 \\
\hline xwords & & 2.380 & 0.930 & 2.559 & 14 \\
\hline TVscripts & $*$ & 3.527 & 2.254 & 1.565 & 14 \\
\hline univs & & 3.634 & 1.507 & 2.411 & 13 \\
\hline childrens & * & 3.631 & 2.045 & 1.776 & 13 \\
\hline blurbs & & 3.399 & 1.363 & 2.494 & 12 \\
\hline $\mathrm{BMJ}$ & & 3.340 & 1.555 & 2.148 & 12 \\
\hline economy & & 2.495 & 1.276 & 1.955 & 12 \\
\hline lawreps & & 2,316 & 0.866 & 2.674 & 10 \\
\hline emails & & 2.399 & 1.025 & 2.340 & 10 \\
\hline tourism & * & 2.685 & 1.815 & 1.479 & 10 \\
\hline footie & & 2.585 & 1.386 & 1.865 & 9 \\
\hline obits & & 2.369 & 0.948 & 2.499 & 8 \\
\hline church & & 2.179 & 1.359 & 1.603 & 8 \\
\hline weather & * & 1.907 & 1.638 & 1.164 & 7 \\
\hline
\end{tabular}

1.65 threshold, suggesting lack of homogeneity among the recipes, the average of the scores for pairwise comparisons with other groups is sufficiently high to compensate this: as Table 6 shows, the average scores for recipes are consistently high, and often the highest in any row. This can be contrasted with the case of the 'church' group, where the within-group average is below the 1.65 threshold, but so are nearly half the scores for pairwise comparisons. But the situation can also be contrasted with the 'TVscripts' and 'childrens' groups: pairwise scores with all the other groujps indicate significant differences, but so does the withingroup average. This means that each TV script or children's story seems significantly different from all the other samples, including the other TV scripts or children's stories. For the 'tourism' group, too, the scores for pairwise comparison are about the same as the within-group score. It so happens that these scores are a bit nearer the threshold, so we get a 10-4 'Sig.' score rather than $14-0$, but the conclusion is the same: the WQsum cannot distinguish these sublanguages.

Finally we have the case of the "lawreps' and 'emails', which are internally homogenous, and can be distinguished from some, but not all of the other groups.

Let us now summarize these observations, and categorize the four types:

A Good result. Homogeneous and distinctive sublanguage: 'xwords', 'univs', 'blurbs', 'BMJ', and 'economy'.
$B_{1}$ Distinctive sublanguage though internally less homogeneous: 'recipes'

$B_{2}$ Coherent sublanguage though not always distinctive: 'lawreps', 'emails'

C Distinctive but not coherent: 'TV scripts', 'childrens'

D Not distinguishable from other groups: 'obits', 'tourism', 'footie', 'church', 'weather'

Not surprisingly, if we look at an ordered list of the individual pairwise scores, we find that the worst scores (Table 8) are mostly between the groups of type $\mathrm{B}_{2}$ and $\mathrm{D}$.

Table 8 Ten worst-scoring pairwise comparisons.

\begin{tabular}{|c|c|c|}
\hline lawreps $\left(B_{2}\right)$ & obits (D) & 0.905 \\
\hline church (D) & obits (D) & 0.915 \\
\hline church (D) & lawreps $\left(\mathrm{B}_{2}\right)$ & 1.020 \\
\hline lawreps $\left(B_{2}\right)$ & weather (D) & 1.086 \\
\hline church (D) & emails $\left(B_{2}\right)$ & 1.139 \\
\hline emails $\left(B_{2}\right)$ & lawreps $\left(B_{2}\right)$ & 1.141 \\
\hline blurbs (A) & $\mathrm{BMJ}(\mathrm{A})$ & 1.204 \\
\hline church (D) & footie (D) & 1.216 \\
\hline footie (D) & weather (D) & 1.290 \\
\hline footie (D) & lawreps $\left(\mathbf{B}_{2}\right)$ & 1.299 \\
\hline
\end{tabular}

This suggests that the WQsum test is able to quantify the similarity of individual groups, as well as to distinguish sublanguages. In this experiment we have taken groups of texts and compared them, but in fact the WQsum algorithm is designed to work on the basis of individual texts. In principle, we could simply take a pair of texts and use the algorithm to determine to what extent they are the same sublanguage. It must be said however that it seems to make more sense to use the test in the comparative manner illustrated here, for example comparing three texts to see which pair is most similar. It also seems important to have a baseline score for an established group of texts belonging to the same sublanguage.

\subsection{Reservations and future directions}

A very short time before the final version of this paper was due to be delivered, a further possibility came to our notice. Tweedie \& Donnelly (1996) describe an alternative, multivariate test using weighted cusums to compare more than two texts. Although we have not had a chance to study this proposal, it claims to give more accurate results than the pairwise application of the WQsum formula that has been reported in this paper. An obvious next step is to try their proposal.

Also, a further step that we might take would be to answer the criticism that the scale of our investigation is too small. The fact that we have taken only three 25sentence samples of each sublanguage obviously means 
that our conclusions must be somewhat limited. An anonymous reviewer commented that "the texts were so different, that it shouldn't be hard at all to discriminate between them". The results in Tables 4 and 6 show that this is not the case at all: the groups that the test failed to distinguish are not necessarily those which to the human eye are most similar (see Table 8), nor are the successfully identified groups necessarily the most dissimilar. Perhaps this finding is not so surprising when we consider that the linguistic features that are used in the test are so superficial: there is no reason to expect that the incidence of words beginning with a vowel, for example, would correlate highly with sublanguage type. And therein lies the real interest of this technique: because the linguistic features are superficial, it seems that there is no intuition that we can appeal to here.

Finally, throughout this paper we have referred to 'sublanguage', and the possibility that our WQsum algorithm can identify different sublanguages. It seems that the algorithm can distinguish texts, but it is by no means clear what aspect of their difference it is capturing. It could for example be merely genre, or some other aspect of sublanguage, that it is capturing though again intuitions are difficult to appeal to because of the superficiality of the linguistic features used. We need to look more closely at the differences between the text pairs it fails to distinguish and those where it succeeds, in order to try to get a feel for what, exactly, the test is capturing. Nevertheless, we feel that it is an interesting avenue to explore, the more so as it seems to be quite unlike the other methods described in this field.

\section{References}

Biber, Douglas. 1988. Variation across Speech and Writing. Cambridge University Press.

Biber, Douglas. 1990. Methodological issues regarding corpus-based analyses of linguistic variation. Literary and Linguistic Computing 5:257-269.

Biber, Douglas. 1995. Dimensions of Register Variation: A Cross-Linguistic Comparison. Cambridge University Press.

Bissell, A. F. 1995a. Weighted cumulative sums for text analysis using word counts. Journal of the Royal Statistical Society A, 158:525-545.

Bissell, Derek. 1995b. Statistical Methods for Text Analysis by Word-Counts. Swansea: European Business Management School, University of Wales.

Burrows, J.F. 1992. Computers and the study of literature. In Christopher S. Butler (ed.) Computers and Written Texts, Oxford: Blackwell, 167-204.
Farringdon, Jill M. 1996. Analysing for Authorship: A Guide to the Cusum Technique. Cardiff: University of Wales Press.

Grishman, Ralph \& Richard Kittredge. 1986. Analyzing Language in Restricted Domains. Hillsdale NJ: Lawrence Erlbaum Associates.

Hilton, Michael L. and David I. Holmes. 1993. An assessment of cumulative sum charts for authorship attribution. Literary and Linguistic Computing 8:73-80.

Holmes, David, I. 1994. Authorship attribution. Computers and the Humanities 28:87-106.

Karlgren, Jussi \& Douglas Cutting. 1994. Recognizing text genres with simple metrics using discriminant analysis. COLING 94: The 15th International Conference on Computational Linguistics, Kyoto, Japan, 1071-1075.

Kessler, Brett, Geoffrey Nunberg \& Hinrich Schütze. 1997. Automatic detection of text genre. 35th Annual Meeting of the Association for Computational Linguistics and 8th Conference of the European Chapter of the Association for Computational Linguistics, Madrid, Spain, 39-47.

Kittredge, Richard \& John Lehrberger (eds) 1982. Sublanguage: Studies of Language in Restricted Semantic Domains. Berlin: de Gruyter.

Morton, Andrew Queen. 1978. Literary Detection: How to Prove Authorship and Fraud in Literature and Documents. London: Bowker.

Potter, Rosanne G. 1991. Statistical analysis of literature: a retrospective on Computers and the Humanities, 1966-1990. Computers and the Humanities 25:401-429.

Sekine, Satoshi. 1997. A new direction for sublanguage NLP. In D. B. Jones \& H. L. Somers (eds) New Methods in Language Processing, London: UCL Press, 165-177.

Smith, M.W.A. 1982. Recent experience and new developments of methods for the determination of authorship. ALLC Bulletin 11:73-82.

Somers, Harold. Forthcoming. Using weighted cusums to evaluate a Lewis Carroll pastiche. To appear in Computers and the Humanities.

Tweedie, Fiona J. \& Christl A. Donnelly. 1996. A multivariate test for the attribution of authorship of $N$ texts. In G. Perissinotto (ed.) Research in Humanities Computing 5, Oxford: Oxford University Press, 243-248.

Ule, L. 1982. Recent progress in computer methods of authorship determination. ALLC Bulletin 10:73-89. 
